\title{
A comparative study of supervised machine learning algorithms for the prediction of long-range chromatin interactions
}

\author{
Thomas Vanhaeren $^{1}$, Federico Divina ${ }^{1}$ (), Miguel García-Torres ${ }^{1}$ (D), Francisco Gómez-Vela ${ }^{1}$ (D), \\ Wim Vanhoof ${ }^{2}$, Pedro Manuel Martínez García $3,4, *$ (i) \\ 1 Division of Computer Science, Universidad Pablo de Olavide, 41013 Sevilla, Spain; vtho@alu.upo.es (T.V.); \\ fdivina@upo.es (F.D.); mgarciat@upo.es (M.G.-T.); fgomez@upo.es (F.G.-V.) \\ 2 Faculty of Computer Science, University of Namur, 5000 Namur, Belgium; wim.vanhoof@unamur.be (W.V.) \\ 3 Centro Andaluz de Biología Molecular y Medicina Regenerativa (CABIMER), CSIC-Universidad de \\ Sevilla-Universidad Pablo de Olavide, 41092 Sevilla, Spain \\ 4 Universidad Isabel I, 09003 Burgos, Spain \\ * Correspondence: pedromanuel.martinez@ui1.es (P.M.M.-G.)
}

\begin{abstract}
The role of three-dimensional genome organization as a critical regulator of gene expression has become increasingly clear over the last decade. Most of our understanding of this association comes from the study of long range chromatin interaction maps provided by Chromatin Conformation Capture-based techniques, which have greatly improved in recent years. Since these procedures are experimentally laborious and expensive, in silico prediction has emerged as an alternative strategy to generate virtual maps in cell types and conditions for which experimental data of chromatin interactions is not available. Several methods have been based on predictive models trained on one-dimensional (1D) sequencing features, yielding promising results. However, different approaches vary both in the way they model chromatin interactions and in the machine learning-based strategy they rely on, making it challenging to carry out performance comparison of existing methods. In this study, we use publicly available 1D sequencing signals to model chromatin interactions in two human cell lines and evaluate the prediction performance of 5 popular machine learning algorithms: decision trees, random forests, gradient boosting, support vector machines and multi-layer perceptron. Our approach accurately predicts long-range interactions and reveals that gradient boosting significantly outperforms the other four algorithms, yielding accuracies of $\sim 95 \%$. We show that chromatin features in close genomic proximity to the anchors cover most of the predictive information. Moreover, we demonstrate that gradient boosting models trained with different subsets of chromatin features, unlike the other methods tested, are able to produce accurate predictions. In this regard, and besides architectural proteins, transcription factors are shown to be highly informative. Our study provides a framework for the systematic prediction of long-range chromatin interactions, identifies gradient boosting as the best suited algorithm for this task and highlights cell-type specific binding of transcription factors at the anchors as important determinants of chromatin wiring.
\end{abstract}

Keywords: machine-learning; chromatin interactions; prediction; genomics; genome architecture

\section{Introduction}

Mammalian genomes stretch for more than 2 meters and are formed by around 3 billion base pairs that are tightly packed within the nucleus, which has a width on the order of micrometers. Strikingly, this level of compaction is compatible with a proper accessibility to the cellular machinery required for essential metabolic processes like replication or transcription. Over the last years, it has become clear that such seemingly counterintuitive events can be explained by the architectural organization of the genome, which forms 3D structures with several levels of complexity $[1,2]$. 
bioRxiv preprint doi: https://doi.org/10.1101/2020.06.09.141473; this version posted June 10, 2020. The copyright holder for this preprint (which was not certified by peer review) is the author/funder. All rights reserved. No reuse allowed without permission.

Beyond nucleosome-nucleosome interactions, chromatin loops represent the smallest scale of genome organization. Loops bring distal genomic loci into close physical proximity and typically range from one to several hundreds of kilobases [3,4]. On a larger scale, chromosomes are spatially segregated into structures called topologically associating domains (TADs). TADs are blocks of chromatin where all pairs of loci interact with each other more frequently than with neighboring regions $[5,6]$. At a higher level, the interactions of TADs with one another make up megabase-scale structures that extend to whole chromosomes and are known as nuclear compartments [7]. Since there is not yet a well characterized biological delineation between such orders of genome organization, in this study we will use the term 'loop' to refer indistinctly to chromatin interactions at any level of this hierarchy. Accordingly, we will refer to the pair of distal loci that are brought together as 'loop anchors'.

Recent findings have revealed that 3D genome organization is more complex than anticipated [3]. Indeed, genome architecture can vary between cell types and is dynamic during cell differentiation and development [8]. Evidence also points to an essential role of the 3D genome in the control of gene expression by allowing communications between promoters and their distal regulatory partners, such as enhancers $[9,10]$. Most of the advances in our understanding of how high order genome organization links to essential cellular metabolic processes comes from the development of Chromatin Conformation Capture (3C)-based technologies [11], which has provided the scientific community with high resolution genome-wide chromatin interaction maps for a number of mammalian cell types. Despite technical improvements, experimental profiling of such maps not only remains difficult and expensive, but also requires a remarkably high sequencing depth for the achievement of high resolution [3,12]. Therefore, in silico predictions that take advantage of the wealth of publicly available sequencing data emerges as a rational strategy to generate virtual chromatin interaction maps in new cell types for which experimental maps are still lacking. To date, several studies have been devoted to predict chromatin loops based on one-dimensional (1D) genomic information with accurate results [13-17]. In such works, authors have modeled loops using different designs and machine learning approaches. Accordingly, they reached different conclusions regarding which chromatin features are most predictive and whether information within the loops (away from the anchors) contributes to the predictive power.

Here, we model chromatin loops using an integrative approach based on ENCODE 1D sequencing datasets to test the performance of 5 different machine learning algorithms: decision trees (DT), random forests (RF), gradient boosting (XGBoost), Support Vector Machines (SVMs) and multi-layer perceptron (MLP). We find that XGBoost achieves the best performance, with precision scores of around 0.95. We also show that, although architectural features at the anchors display the greatest predictive power, transcription-associated features accurately predict chromatin loops. Among these features, transcription factors are the most informative and their contribution resides mainly at the anchors and varies across human cell lines.

\section{Materials and Methods}

\subsection{Processing publicly available data}

The experimental data used in this study is summarized in Table S1. With the exception of Hi-C, which was used for visualization purposes, the rest of the datasets were batch-downloaded from ENCODE [18] . BAM files were used for ChIP-seq, RNA-seq and DNase-seq, while tsv tables labeled as long range chromatin interactions were used for ChIA-PET experiments targeting RAD21. All the analyses were performed in K562 and GM12878 cell lines using human assembly hg19.

\subsection{Identification of RAD21-associated loops}

RAD21 loops in K562 were extracted from ENCODE dataset ENCFF002ENO. For the identification of RAD21 loops in GM12878, two replicates were considered (datasets ENCFF002EMO and ENCFF002EMQ) and only overlapping loops were retained. We defined overlapping loops as those 
bioRxiv preprint doi: https://doi.org/10.1101/2020.06.09.141473; this version posted June 10, 2020. The copyright holder for this preprint (which was not certified by peer review) is the author/funder. All rights reserved. No reuse allowed without permission.

that share both anchors, allowing a maximum gap of $2 \mathrm{~kb}$. Following this approach, we identified 3,290 and 5,486 loops in K562 and GM12878, respectively. For machine learning classification, we generated the same number of negative loops for each cell line. In order to build robust models that were able to accurately separate loops from random genomic regions, we generated an initial set of negative loops by randomly combining pairs of RAD21 ChIP-seq peaks (see Figure 2a ). Then, loops that overlapped experimental ones were filtered out. Since genomic distance highly influences chromatin interactions, the resulting set of negative loops were passed through a regression model trained to capture the distribution of genomic distances between anchors of experimental loops. Finally, 3, 290 and 5, 486 loops were randomly selected from the K562 and GM12878 negative sets, respectively. In this way, the number of positive and negative loops in the final datasets were equal and with similar genomic distance distributions.

\subsection{Machine learning data matrix}

In order to model loops using chromatin features, we quantified 23 sequencing datasets within and adjacent to each loop using a modified version of the approach described by Handoko et al. [19]. Given a loop with length $\mathrm{L}$, we extended $\mathrm{L}$ base pairs to its left and right and the extended region (with length 3L) was splitted into 1500 bins (Figure 2b, top). Then, we scored the sequencing experiments (Table S1) within each bin (Figure 2b, bottom). The scoring was performed by counting reads that aligned to each bin and normalizing by bin genomic length and sequencing library size. For each cell line, we obtained a final data matrix with rows representing loops and columns representing quantification of chromatin features at each bin.

The 23 chromatin feature datasets (Table S1) included marks associated to chromatin accessibility (DNAse-seq), expression (RNA-seq), RNA Pol2 binding (POLR2A, POLR2AphosphoS5), active promoters (H3k4me2, H3k4me3, H3k9ac), enhancers (H3k4me1), active gene bodies and elongation (H3k36me3, H4k20me1), transcriptional repression (H3k27me3, H3k9me3), architectural components (CTCF, RAD21) and transcription factors (ATF3, CEBPB, JUND, MAX, REST, SIN3A, SP1, SRF, YY1).

\subsection{Supervised learning}

In this work, we have used five Machine Learning strategies in order to induce a classification model for the data. Each of the model is applied to a training set and then the prediction models are evaluated on a test set. We briefly describe each method in the following subsections.

\subsubsection{Decision Trees (DT)}

We have used the algorithm implementation provided by [20,21]. A decision tree (DT) algorithm iteratively builds a classification tree by adding a node to the tree. Features are used within internal nodes in order to classify examples.

A DT algorithm induces a tree by splitting the original dataset into smaller sets based on a test applied to the features. This process is repeated recursively on each smaller set and is complete when the small set present in one node presents the same value as the target label or when no more gain in predictive power is obtained by splitting further. Such a process is known as recursive partitioning.

The features selected depend on a measure used in order to assess the importance of a feature. The Gini Impurity measure was used, which is defined as:

$$
1-\sum_{i=1}^{n} p^{2}\left(c_{i}\right)
$$

where $p\left(c_{i}\right)$ s the probability of class $c_{i}$ in a node. We have also run several experiments on the datasets used in the paper with the Entropy, and the results were basically the same with the two measures.

In this paper we have used the implementation provided by the library Scikit Learn (Sklearn) library [20]. The maximum depth parameter has been set to 10. The criterion was set to Entropy, while 
bioRxiv preprint doi: https://doi.org/10.1101/2020.06.09.141473; this version posted June 10, 2020. The copyright holder for this preprint (which was not certified by peer review) is the author/funder. All rights reserved. No reuse allowed without permission.

Gini is in theory intended for continuous attributes, Entropy is intended for attributes occurring in classes but since Entropy uses logarithmic functions slower to compute. For the training of the K562 DT, both criteria were tested and compared.

\subsubsection{Random Forests (RF)}

This strategy [22] belongs to the family of ensemble learning algorithms, based on a divide and conquer approach that improves performance. The principle behind ensemble methods is that a group of weak models are put together to form a stronger model. This is due to the fact that ensemble strategies reduce variance, improving the prediction power. RF algorithms induce a set of trees, which are then used in order to produce the final output, using a voting scheme. The trees are built one at a time. Each tree is obtained using a randomly selected training subset and a randomly selected subset of features. It follows that the trees depend on the values of an independently sampled input data set, using the same distribution for all trees.

In this case we have also used the implementation provided in [21]. We have set the number of estimators to 250, even if we have noticed that the results show little variation with estimators between 50 and 250. The maximum depth of the trees was set to 8, and the criteria used was the Entropy.

\subsubsection{XGBoost}

This algorithm, as well as other gradient boosting algorithms like the well known Gradient Boosting Method (GBM), sequentially assembles weak models to produce a predictive model [23]. During this sequential procedure, a gradient descend procedure is applied. This procedure is repeated until a given number of trees has been induced, or when no improvement is registered. An important aspect of XGBoost is how the algorithm controls over-fitting, which is a known issue in gradient boosting algorithms. XGBoost adopts a more regularized model formalization, which allows the algorithm to obtain better results than GBM. XGBoost is a method that has recently received much attention in the data science community, as it has been successfully applied in different domains. This popularity is mostly due to the scalability of the method. In fact, XGBoost can run up to ten times faster than other popular approaches on a single machine. Again, we have used the Scikit-learn library for this algorithm. In this case the number of estimators was set to 100, the learning rate to 0.1 and the maximum depth to 3 .

\subsubsection{Artificial Neural Networks (ANNs)}

In a rough analogy with biological learning system, ANNs consist of densely interconnected units, called neurons [24]. Each neuron receives a number of real valued input, e.g., from other neurons of the network, and produces a single real valued output. The output depends on an activation function used in each unit, which introduces non-linearity to the output. The activation function is used only if the input received by a unit is higher than a given activation threshold. If this is not the case, then no output is produced. Normally, an ANN consists of different layers of neurons. Among such layers we can distinguish the input and output layers, and in between there may be one or more hidden layers.

In this paper we have used the Multi-layer Perceptron (MLP) classifier provided by Sklearn library [20]. In particular, for the experiments performed on the K562 cellular line, we have used an Alpha (regularization term) of 0.001 , with an adaptive learning rate and a network consisting of 40 layers with 10 neurons on the first layer. Alpha forces the parameters to have small values for robustness. The optimal hyper-parameters used to train the MLP classifier for the GM12878 cell dataset were the same as for the K562 cell, except for the Hidden Layer Sizes which was set to 100 layers, with 20 neurons on the first layer. These parameters were experimentally determined.

\subsubsection{Support Vector Machines (SVM)}

This supervised technique tries to find an hyperplane in a $n$-dimensional space (being $n$ the number of features), capable of separating the training examples [25]. Several such hyperplanes may 
bioRxiv preprint doi: https://doi.org/10.1101/2020.06.09.141473; this version posted June 10, 2020. The copyright holder for this preprint (which was not certified by peer review) is the author/funder. All rights reserved. No reuse allowed without permission.

exist, and SVM aims at finding the one that maximazes the distance between data points of the classes. The hyperplane is basically the classification model, with data falling on one side of the hyperplance being assigned a class, while data falling on the other side are assigned the other class. Data are not always linearly separable, and in these cases, a kernel function is used in order to transform the original data points with the aim of mapping the original data points into a higher dimensional space where we can find a hyperplane that can separate the samples.

In this study we have used the Radial Basis Function (RBF) kernel. The RBF kernel is an often used kernel for classification tasks [26]. In particular, the C-value for the RBF function was set to 100, after having performed several preliminary experiments.

In order to evaluate the methods, we have used four popular measures [27]:

$$
\begin{gathered}
\text { Accuracy }=\frac{T P+T N}{T P+T N+F P+F N} \\
\text { Precision }=\frac{T P}{T P+F P} \\
\text { Recall }=\frac{T P}{T P+F N} \\
F 1-\text { Score }=\frac{2 \times \text { Precision } \times \text { Recall }}{\text { Precision }+ \text { Recall }}
\end{gathered}
$$

In the above equation we have used the following terminology:

- True Positive (TP): a true positive represents the case when the actual class matches the predicted class;

- True Negative (TN): a true negative is similar to a true positive, the only difference being that the actual and predicted classes are part of the negative examples;

- False Positive (FP): a false positive is the case when the actual class negative, but the example is classified as positive;

- False Negative (FN): similar to false positive, but it this case this refers to the case where a positive example is classified as negative.

\section{Results and Discussion}

\subsection{Association of genomic and epigenomic features with chromatin loops}

We started by assessing the genomic and epigenomic landscape of chromatin loops. We used published ChIA-PET datasets from two human cancer cell lines (Table S1), K562 and GM12878, identifying 3,290 and 5,486 RAD21-mediated chromatin loops, respectively. 1D sequencing datasets from ENCODE (ENCODE Project Consortium, 2012) were also collected in order to represent the chromatin features associated to loops and their genomic neighbourhoods (Figure 1, Figure S1). As expected, we observed a large colocalization of CTCF and RAD21 architectural proteins with loop anchors, in agreement with previously reported data $[5,28]$. On the other hand, whereas the repressive mark H3K27me3 and the gene body mark H3K36me3 were found to be dispersed along loops and across loop anchors, other regulatory marks were enriched both at loop anchors and inside the loops. Such is the case of Pol2, open chromatin measured by DNase-seq and several transcription factors. The same stands for H3K4me1 and H3K4me3, two well-known markers for enhancers and promoters. These observations suggest that regulatory features measured by high-throughput sequencing provide a valuable source of information for the prediction of chromatin loops.

\subsection{An integrative approach to predict chromatin loops}

In order to integrate sequencing features data into a predictive model of chromatin loops, we applied the computational framework of Figure 2. We first generated positive and negative sets of 


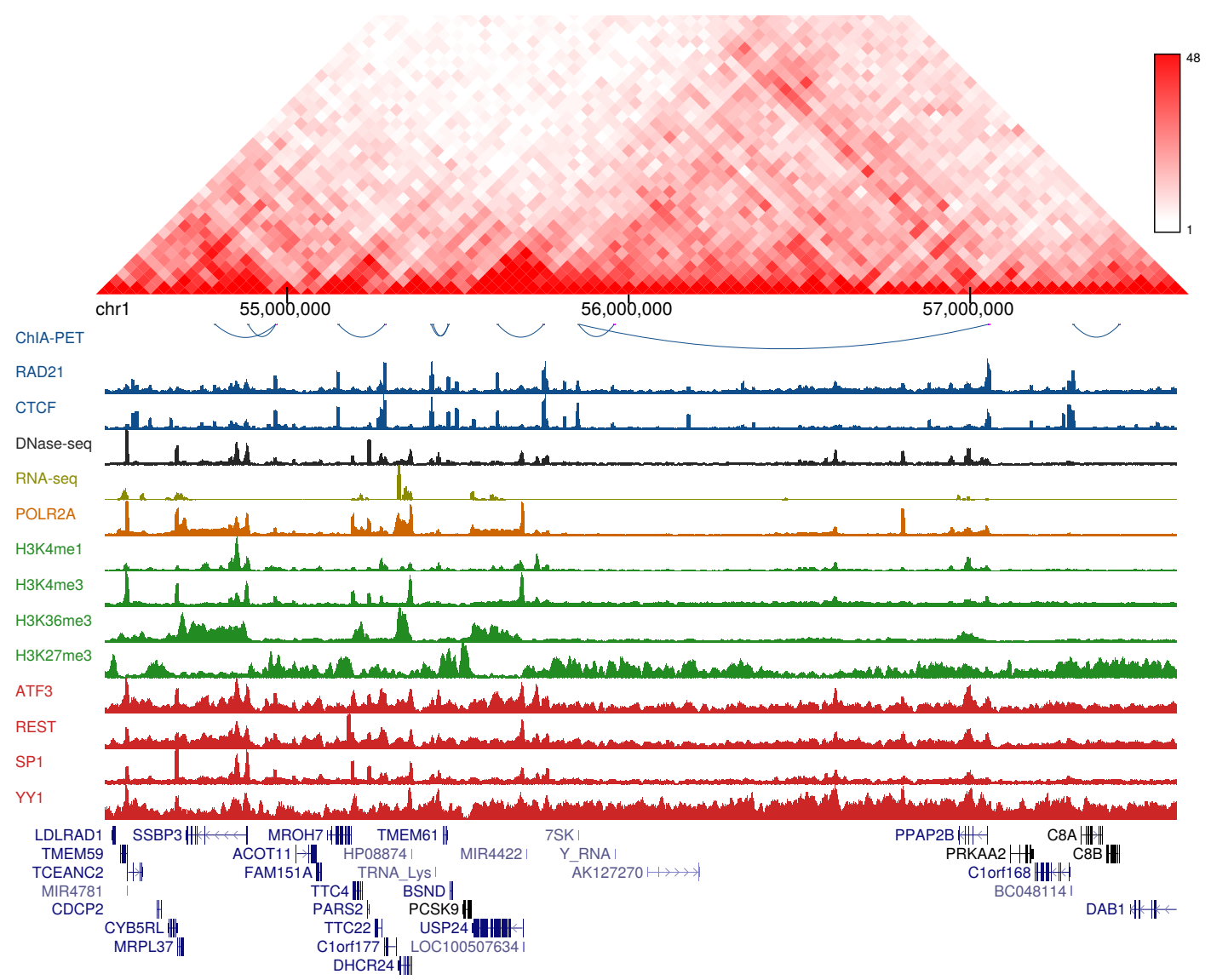

Figure 1. Chromatin features associated with RAD21 loops in GM12878 cell line. Hi-C interaction frequencies are shown in the top panel and ChIA-PET interactions are represented as blue arcs in the second panel. Then, a genome browser view for relevant chromatin features is displayed, including architectural factors (blue), DNase-seq (black), RNA-seq (yellow), RNA Pol2 (orange), histone marks (green) and transcription factors (red).

RAD21-associated chromatin loops. We used the experimental loops from the previous section as the positive set and the negative set was generated by combining pairs of RAD21 ChIP-seq peaks from ENCODE that do not overlap experimental loop anchors (see Material and Methods). Given the relative distribution of regulatory marks with respect to chromatin loops (Figure 1), we argued that these marks may affect loops not only at the anchors but also at the region between them. Therefore, to comprehensively measure the occupancy of chromatin features within and adjacent to each loop, we based our strategy on the approach of [19]. Given a loop with length L, we extended L base pairs to its left and right and the extended region (with length 3L) was partitioned into 1500 bins. For each bin, 23 high-throughput sequencing experiments (Table S1) were scored, resulting in a data matrix with rows representing loops and columns representing the scored experiments at each bin. Finally, we trained and tested classifiers in both cell lines using five machine learning algorithms. This design based on multi bins allowed us to measure the position-specific ability of sequencing data to predict chromatin loops with an unprecedented resolution. As a result, we ended up with model matrices of 34, 500 columns and either 3, 290 (K562) or 5, 486 (GM12878) rows (Figure 2).

\subsection{Model performance}

The final feature matrices were divided into training (80\%) and test (20\%) and 5 classification algorithms were applied: decision trees, random forests, XGBoost, SVM and MLP. To evaluate the performance of classification, trained models were applied to the test sets and several metrics were calculated including accuracy (Acc), precision, recall and F1-score. Accurate predictions were obtained 
bioRxiv preprint doi: https://doi.org/10.1101/2020.06.09.141473; this version posted June 10, 2020. The copyright holder for this preprint (which was not certified by peer review) is the author/funder. All rights reserved. No reuse allowed without permission.

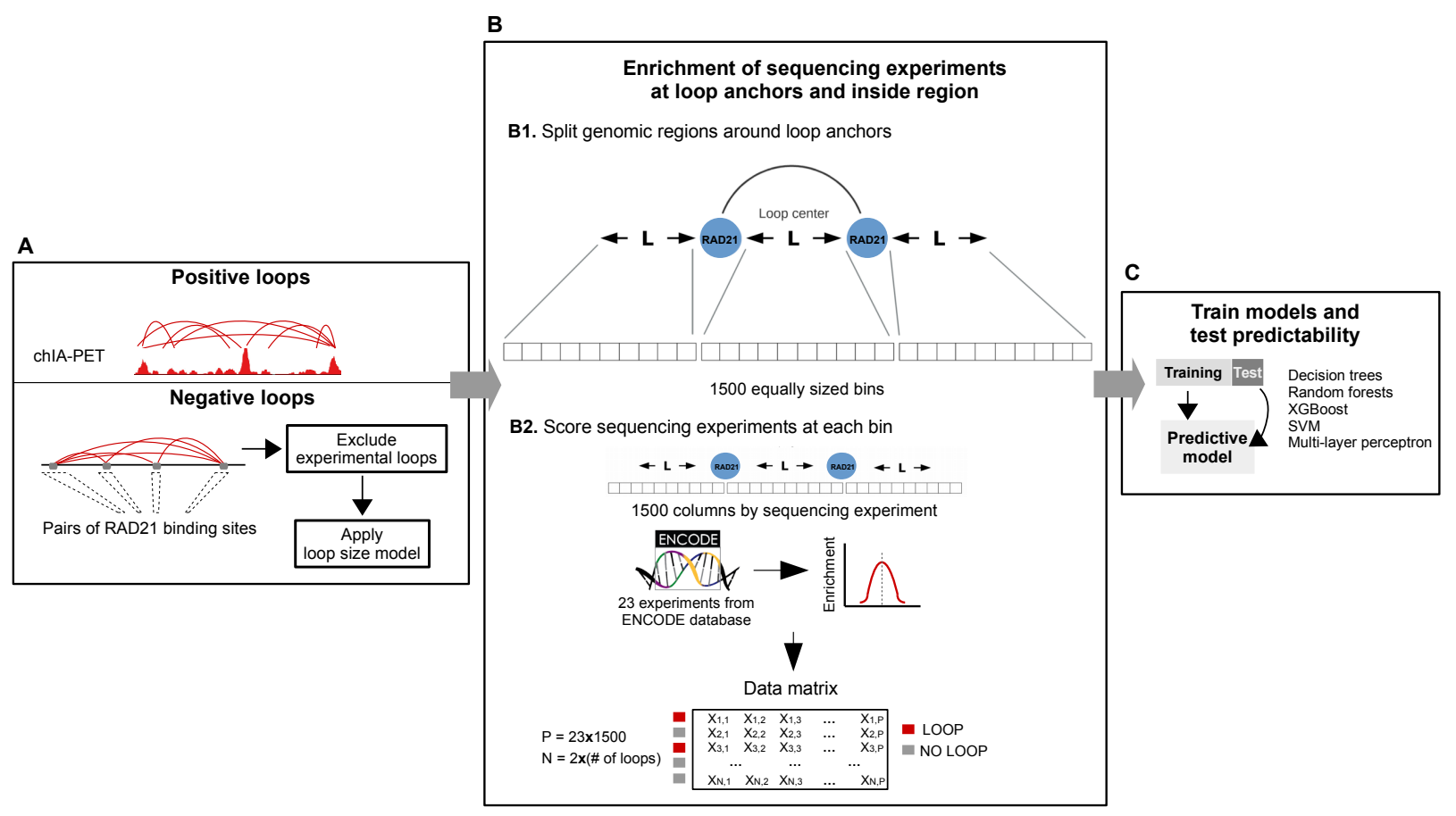

Figure 2. Illustration of the integrative machine learning schema for the prediction of chromatin loops. A. Positive and negative RAD21-associated loops were first identified (see Methods). B. Then, given a loop with length $\mathrm{L}$, we extended L base pairs to its left and right and the extended region (with length 3L) was partitioned into 1500 bins. For each bin, 23 high-throughput sequencing experiments were scored, resulting in a data matrix with rows representing loops and columns representing the scored features. C. Finally, we trained and tested classifiers using five machine learning algorithms.

by the 5 algorithms, with almost no differences on the performance metrics and with similar values in both cell lines (Tables 1 and 2). The smallest accuracies were obtained by SVM, with values of 0.8087 and 0.8219 for GM12878 and K562, respectively. On the other hand, XGBoost significantly outperformed the rest of the methods, achieving accuracies of 0.9474 and 0.9634 for GM12878 and K562, respectively. As a matter of fact, the other three classification algorithms achieved very similar performances for GM12878 cell line, with Acc of around 0.83, while for K562 they ranged from 0.8226 to 0.8698 . These results demonstrate that chromatin loops can be accurately predicted using our integrative approach and indicate that gradient boosting by XGBoost provides the best performance for this task.

Table 1. Machine learning performance of the proposed models in K562 cell line.

\begin{tabular}{lllll}
\hline Algorithm & Accuracy & Precision & Recall & F1-Score \\
\hline Decision Trees & 0.8698 & 0.8707 & 0.8699 & 0.8698 \\
Random Forests & 0.8424 & 0.8469 & 0.8425 & 0.8419 \\
XGBoost & 0.9634 & 0.9638 & 0.9635 & 0.9635 \\
SVM & 0.8219 & 0.8224 & 0.8219 & 0.8218 \\
MLP & 0.8226 & 0.8231 & 0.8227 & 0.8226 \\
\hline
\end{tabular}


bioRxiv preprint doi: https://doi.org/10.1101/2020.06.09.141473; this version posted June 10, 2020. The copyright holder for this preprint (which was not certified by peer review) is the author/funder. All rights reserved. No reuse allowed without permission.

3.4 Loop anchors are the most informative regions

Table 2. Machine learning performance of the proposed models in GM12878 cell line.

\begin{tabular}{lllll}
\hline Algorithm & Accuracy & Precision & Recall & F1-Score \\
\hline Decision Trees & 0.8313 & 0.8314 & 0.8313 & 0.8313 \\
Random Forests & 0.8262 & 0.8284 & 0.8263 & 0.8261 \\
XGBoost & 0.9474 & 0.9485 & 0.9475 & 0.9474 \\
SVM & 0.8087 & 0.8088 & 0.8088 & 0.8087 \\
MLP & 0.8322 & 0.8328 & 0.8323 & 0.8322 \\
\hline
\end{tabular}

\section{Decision trees}

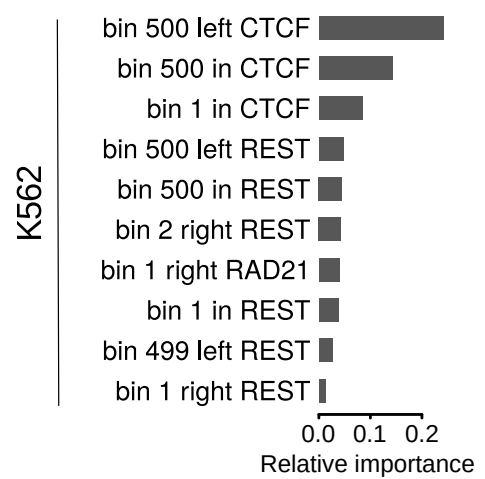

Random forests
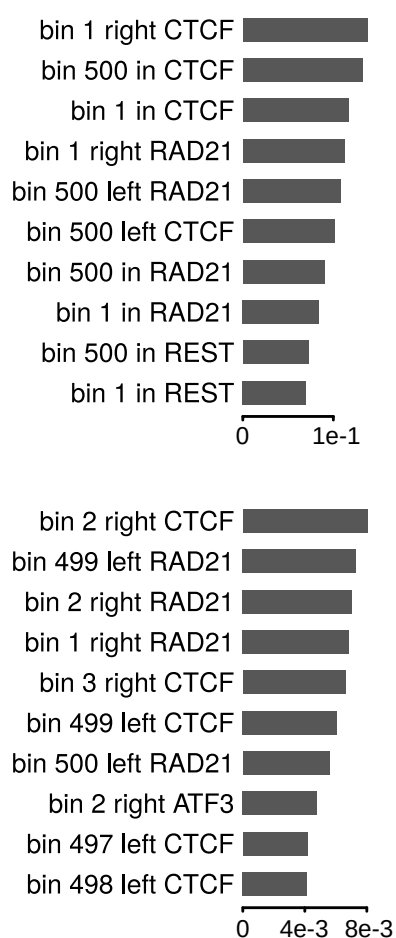

XGBoost

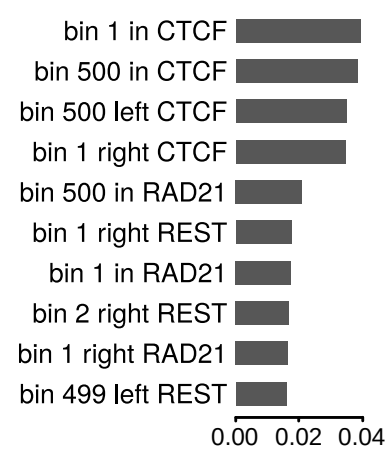

bin 4 right $\mathrm{CTCF}$ bin 497 left RAD21 bin 499 left RAD21 bin 496 left CTCF bin 1 in CTCF bin 2 right RAD21 bin 500 in CTCF bin 1 right DNase-seq bin 500 left DNase-seq bin 500 in RAD21 $0 \longdiv { 1 . 5 e - 2 }$

Figure 3. Ranking of top 10 important features for the prediction of RAD21 chromatin loops. Horizontal bars represent relative importances of featured bins. The terms 'left', 'in' and 'right' are used for bins from 1 to 500,501 to 1000 and 1001 to 1500 , respectively. The relative position of the bins within one of these 3 windows is also included in the feature names.

\subsection{Loop anchors are the most informative regions}

Next, we explored the most informative features for predicting chromatin loops. The design of our approach based on Handoko et al. [19] represents each chromatin feature as a 1,500-bin array (Figure 2), which allowed us to comprehensively evaluate the contribution of a given feature according to its relative position within and at both sides of loops. Among the five methods we compared, DT, $\mathrm{RF}$ and XGBoost assign an importance measure during the training process, providing information on which repertoire of features are the most relevant in the classification. For this reason, and given that these algorithms showed better overall performances than SVM and MLP (Tables 1 and 2), the latter were excluded from this analysis. As expected, binding of architectural components (CTCF, RAD21) showed the highest predictive power in both cell lines and regardless the learning algorithm (Figures 3 and S2). We observed that the relative positions of the most informative features are highly biased towards genomic bins close to loop anchors. This is true for the top 10 important bins of both cell lines and for the three tested algorithms, suggesting that chromatin information between anchors in only modestly predictive. 
bioRxiv preprint doi: https://doi.org/10.1101/2020.06.09.141473; this version posted June 10, 2020. The copyright holder for this preprint (which was not certified by peer review) is the author/funder. All rights reserved. No reuse allowed without permission.

Although the three methods overall agreed on the top important features, while for DT the predictive power is concentrated in $\sim 10$ bins, this is not the case of RF and XGBoost, where it seemed to be more widely distributed (Figure S2). Among the top 10 important features of each algorithm in GM12878, bins associated with CTCF and RAD21 binding around loop anchors were largely predominant (Figure 3, bottom panel). The only exception was a bin associated to the transcription factor ATF3 at the right anchors, which was identified as the 8th most important feature by RF. On the other hand, in K562 REST was found together with CTCF and RAD21 among the top 10 important features by the three methods (Figure 3, top panel). Bins associated to this transcriptional repressor at loop anchors, although less informative, are as frequent as those associated to CTCF and RAD21.

The contribution of features that do not belong to the top 10 important ones greatly varies from one method to another. For example, DT and XGBoost reported several bins associated to histone marks among the top 30 important features in both cell lines, while this was never the case for RF (Figure S2). On the other hand, RF seemed to assign some predictive importance to DNase-seq and, in a lesser extent, to YY1, whereas these features were rarely found among the top 30 important features when using DT and XGBoost. These observations are likely to indicate that the contribution of such features is negligable compared to the top important ones.

Our design also allowed us to represent the contribution of each chromatin feature according to its relative position within and at both sides of loops (Figures 5, 4 and S3A-F). In agreement with the importance analysis, most of the predictive information for the majority of the evaluated chromatin datasets resides at the anchors and their close genomic vicinity. Besides the architectural components CTCF and RAD21, this is particularly prominent for DNase I hypersensitivity and transcription factors (Figures 5 and 4). Altogether, these results suggest that, in addition to architectural components, transcriptional features at the anchors contribute to chromatin wiring in RAD21-mediated loops.
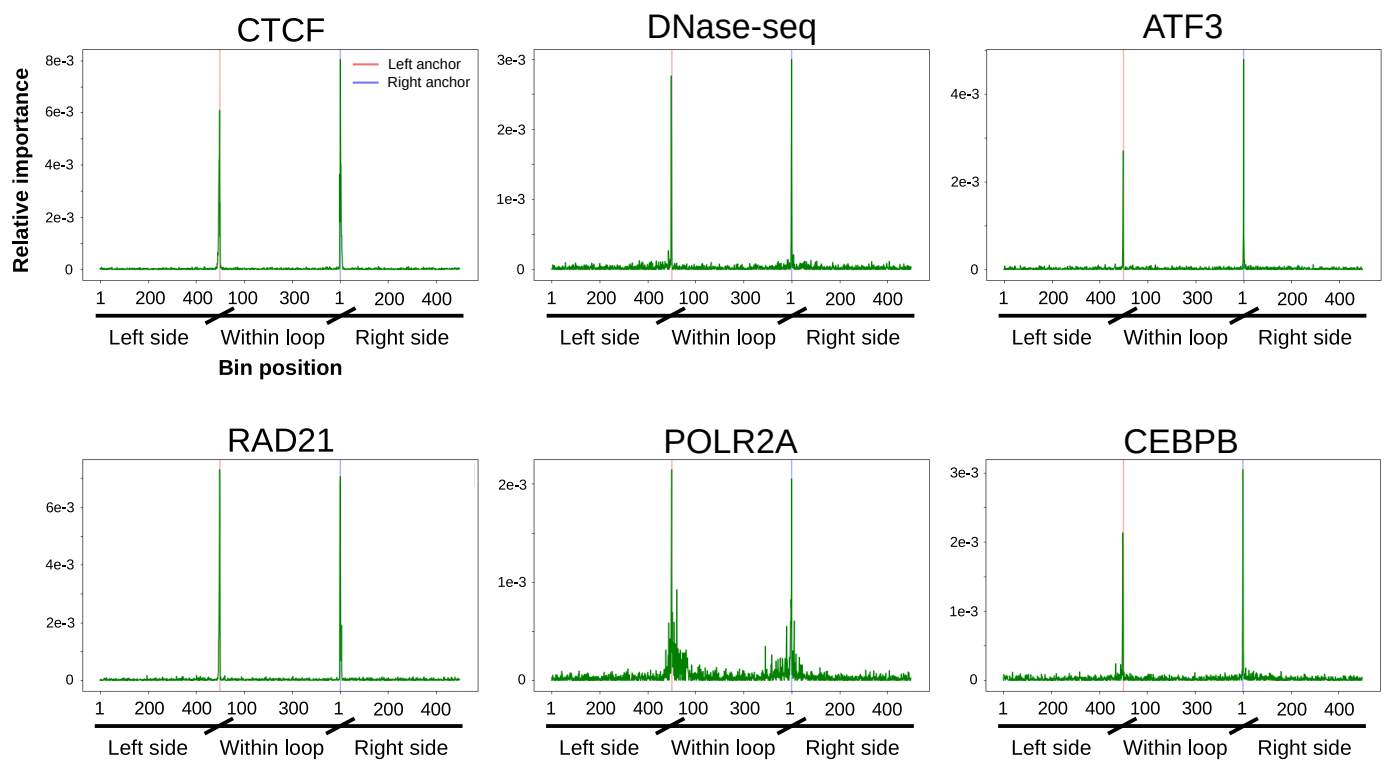

Figure 4. Position specific importance of selected high-throughput sequencing datasets in GM12878 cell line. Random forests importance score is shown for the 1500 bins corresponding to the most informative experiments. Coordinates of the $\mathrm{x}$-axis are similar to those of Figure 5. Figures S3D-F display similar plots for all the tested datasets as well as for Decision Trees and XGBoost algorithms. 
bioRxiv preprint doi: https://doi.org/10.1101/2020.06.09.141473; this version posted June 10, 2020. The copyright holder for this preprint (which was not certified by peer review) is the author/funder. All rights reserved. No reuse allowed without permission.
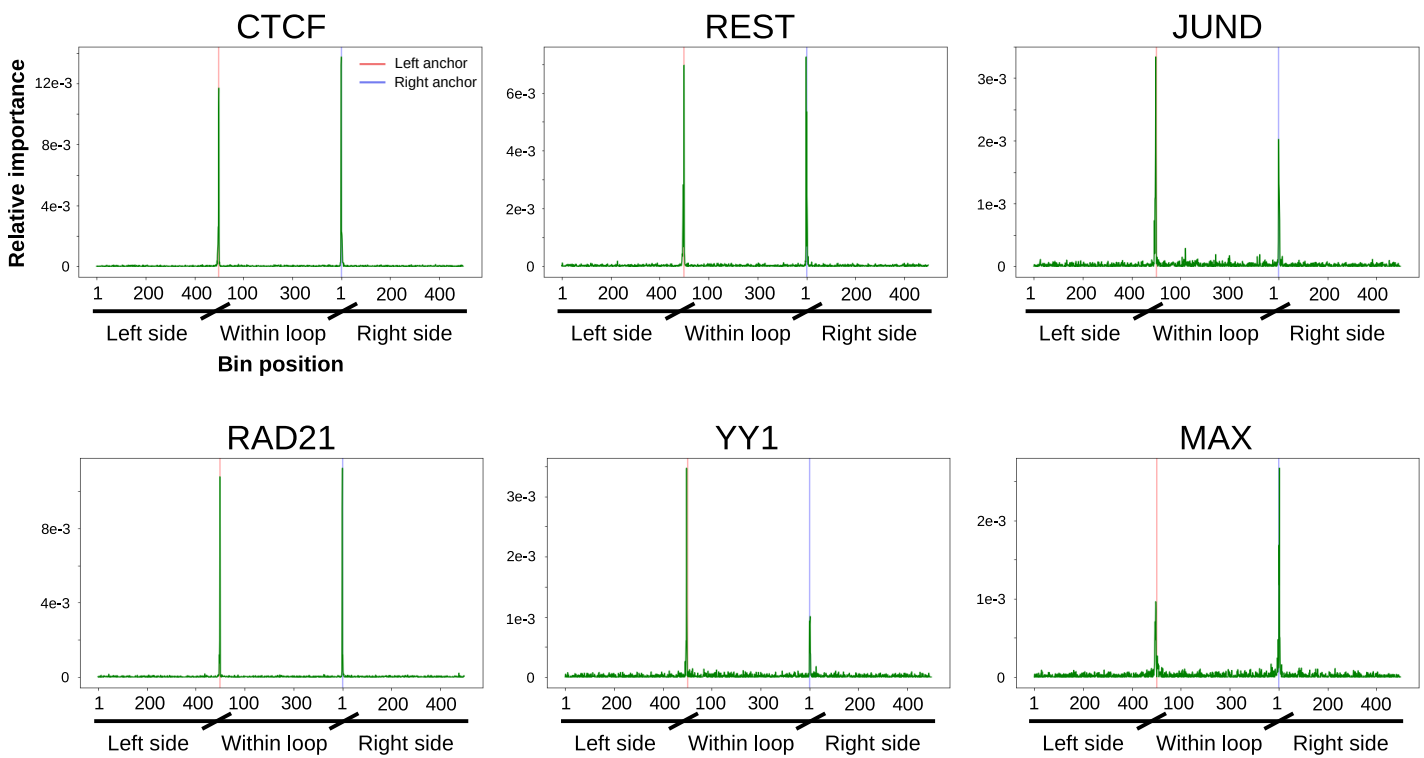

Figure 5. Position specific importance of selected high-throughput sequencing datasets in K562 cell line. Random forests importance score is shown for the 1500 bins corresponding to the most informative experiments. Coordinates of the $\mathrm{x}$-axis are similar to those described in Figure 3, with left and right anchor position represented as red and blue vertical lines, respectively. Figures S3A-C display similar plots for all the tested datasets as well as for Decision Trees and XGBoost algorithms.

\subsection{Removing architectural features has a modest effect on XGboost performance for K562}

Given the importance of transcription associated factors in the final predictions, we next investigated whether these features alone can be used in order to predict chromatin loops. To this aim, we removed CTCF and RAD21 from the matrices and evaluated models trained with the rest of the datasets. We observed that the 5 algorithms decreased their performances, with significant differences in the acquired predictions and GM12878 showing more pronounced drops (Tables 3 and 4). The highest decrease was reported by DT in GM12878 cell line, which yielded an accuracy of 0.68 , while when the whole set of features was used, the accuracy achieved was of 0.83 (Table 2). SVM and MLP performances in both cell lines also seemed to be drastically affected by the removal of architectural factors. While these methods exhibited accuracies of 0.81-0.83 in the previous analysis, these values decreased to 0.70-0.73 after removing CTCF and RAD21 ChIP-seq data. Although its performance sensibly decreased in GM12878, XGboost was found again to achieve the best predictions. Strikingly, the performance of this algorithm was almost not affected by the removal of architectural components in K562 cell line, yielding an accuracy of 0.95 (Table 3). This result agrees with our previous analysis of relative importance, in which the binding of REST transcription factor at loop anchors was found to be among the most predictive features. We can then conclude that, at least in K562, transcription information alone is enough for the prediction of RAD21-mediated chromatin interactions.

Exploration of the most informative features in the new DT, RF and XGBoost models reveals that most of the predictive power resides in transcription factor binding and, to a lesser extend, DNase I hypersensitivity and RNA polymerase II (Pol II) binding (Figures 6 and S4). Again, we observed that the relative positions of these features were biased towards genomic bins in close proximity to loop anchors. Although different prediction accuracies were achieved by the three algorithms, overall the sets of top important features they provided were similar. Since XGBoost yielded the best performance, we focused on the importances reported by this algorithm. We observed that different transcription factors govern the contribution of the two cell lines. While bins associated with REST and MAX were predominant among the top 10 features in K562 (Figure 6, top right), this was not the case in GM12878, for which ATF3 and CEBPB (together with DNAse-seq and Pol II) were found to contribute most to the predictions (Figure 6, bottom right). Given the role that chromatin interactions play in 
bioRxiv preprint doi: https://doi.org/10.1101/2020.06.09.141473; this version posted June 10, 2020. The copyright holder for this preprint (which was not certified by peer review) is the author/funder. All rights reserved. No reuse allowed without permission.

\subsection{XGboost models achieve accurate predictions when trained with subsets of chromatin features 6}

gene regulation, these results agree with distinct gene regulatory programs being maintained through cell-type specific binding of transcription factors [29,30], and highlights transcriptional features as important determinants of chromatin wiring mediated by RAD21.

Table 3. Machine learning performance of models trained without architectural factors information in K562 cell line.

\begin{tabular}{lllll}
\hline Algorithm & Accuracy & Precision & Recall & F1-Score \\
\hline Decision Trees & 0.8257 & 0.8259 & 0.8257 & 0.8257 \\
Random Forests & 0.7846 & 0.7877 & 0.7846 & 0.7840 \\
XGBoost & 0.9467 & 0.9469 & 0.9467 & 0.9467 \\
SVM & 0.7264 & 0.7264 & 0.7237 & 0.7228 \\
MLP & 0.7168 & 0.7202 & 0.7169 & 0.7157 \\
\hline
\end{tabular}

Table 4. Machine learning performance of models trained without architectural factors information in GM12878 cell line.

\begin{tabular}{lllll}
\hline Algorithm & Accuracy & Precision & Recall & F1-Score \\
\hline Decision Trees & 0.6806 & 0.6817 & 0.6806 & 0.6805 \\
Random Forests & 0.7626 & 0.7648 & 0.7627 & 0.7624 \\
XGBoost & 0.8327 & 0.8334 & 0.8327 & 0.8327 \\
SVM & 0.7046 & 0.7068 & 0.7046 & 0.7042 \\
MLP & 0.7018 & 0.7061 & 0.7018 & 0.7008 \\
\hline
\end{tabular}
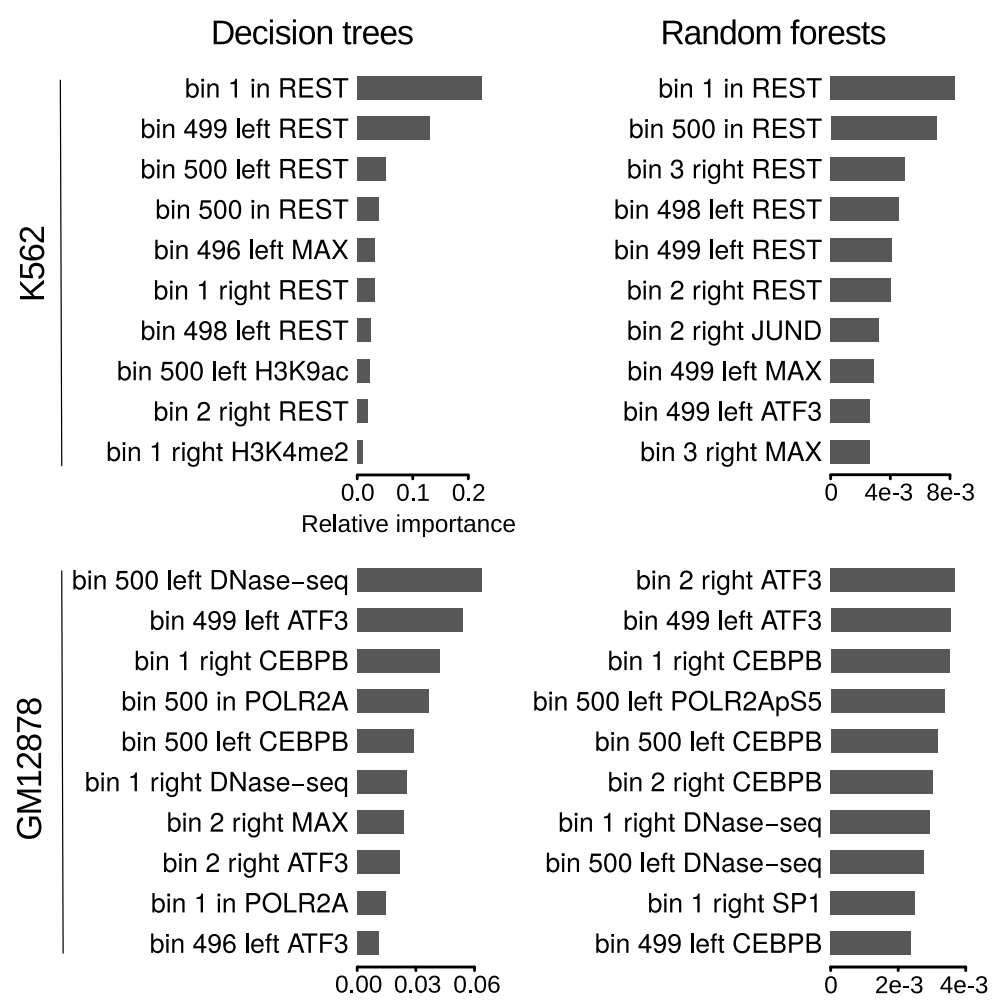

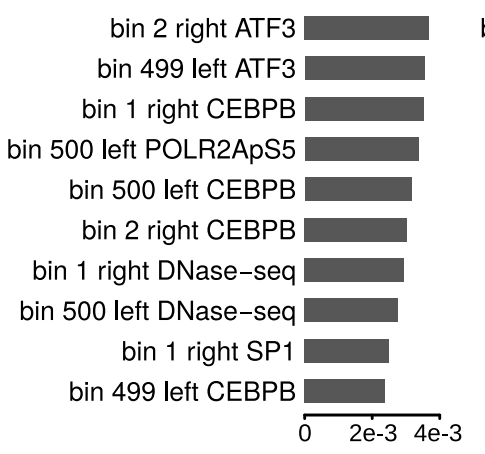

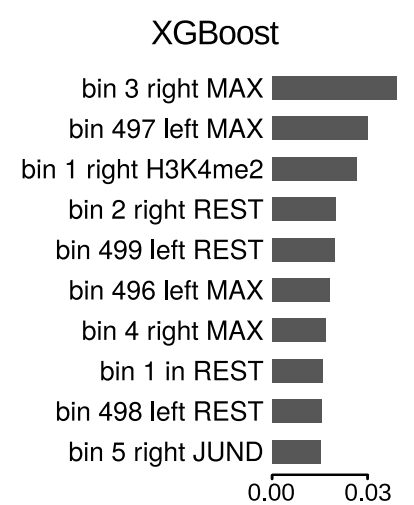

bin 500 left POLR2ApS5

bin 500 left POLR2A

bin 500 left DNase-seq

bin 500 in POLR2A

bin 1 in POLR2A

bin 499 left ATF3

bin 500 left CEBPB

bin 1 in POLR2ApS5

bin 1 right DNase-seq

bin 1 right POLR2ApS5

0.000 .01

Figure 6. Ranking of top 10 important features for the prediction of RAD21 chromatin loops using only features associated with transcription. Horizontal bars represent relative importances as in Figure 3. 
bioRxiv preprint doi: https://doi.org/10.1101/2020.06.09.141473; this version posted June 10, 2020. The copyright holder for this preprint (which was not certified by peer review) is the author/funder. All rights reserved. No reuse allowed without permission.

XGboost models achieve accurate predictions when trained with subsets of chromatin featBiwe \$6

\subsection{XGboost models achieve accurate predictions when trained with subsets of chromatin features}

Since the removal of architectural components yielded different outcomes depending both on the algorithm type and the cell line, we next explored to what extent specific subsets of chromatin features provide different prediction abilities. To that aim, we trained and tested DT, RF and XGBoost models based on the following categories: Architectural (only bins associated with CTCF and RAD21), TF (bins associated with transcription factors ATF3, CEBPB, JUND, MAX, REST, SIN3A, SP1, SRF and YY1), Architectural-anchors (CTCF and RAD21 around loop anchors) and TF-anchors (TF around loop anchors). To only account for information around anchors, we restricted the model matrices to 100 bins centered at left and right anchors, respectively, obtaining 200 bins (out of the total 1,500 bins; Figure 2b) for each chromatin feature belonging to the defined categories. As a matter of fact, we also included in the analysis the two categories explored in previous sections, which we named All (all bins, as in sections 3.3 and 3.4) and Transcription (TF + DNase-seq + RNA-seq + histone marks, as in section 3.5).

For all the evaluated subsets, XGBoost yielded the best performances in both cell lines (Figure 7 and Tables 1, 2, 3, 4, S2-S9), in agreement with our previous observations. We also observed that models trained with subsets restricted to bins around anchors achieved performances at least as accurate as those obtained using also bins within the loops, which confirms that genomic information away from the anchors poorly contributes to chromatin wiring prediction. Overall, different accuracies were observed for the grouped categories, with GM12878 models being more sensible to subset selection. In this sense, DT and XGBoost showed a similar behaviour (Figure 7, left and right panels; Tables 2, 4, S3, S5, S7, S9). For both algorithms, performance of K562 models were found to be somehow stable across categories, achieving accuracies of 0.83-0.87 (DT) and 0.94-0.96 (XGBoost) (Figure 7, left and right panels; Tables 1, 3, S2, S4, S6, S8). On the other hand, GM12878 models achieved greatly variable predictions for different subsets of features, with TF associated categories yielding the worst performances (0.66-0.68 and $\sim 0.79$ for DT and XGBoost, respectively). Conversely, Architectural categories were found among the most predictive ones, with accuracies of $\sim 0.85$ (DT) and $\sim 0.93$ (XGBoost) (Figure 7, left and right panels; Tables 2, 4, S3, S5, S7, S9).

Decision trees

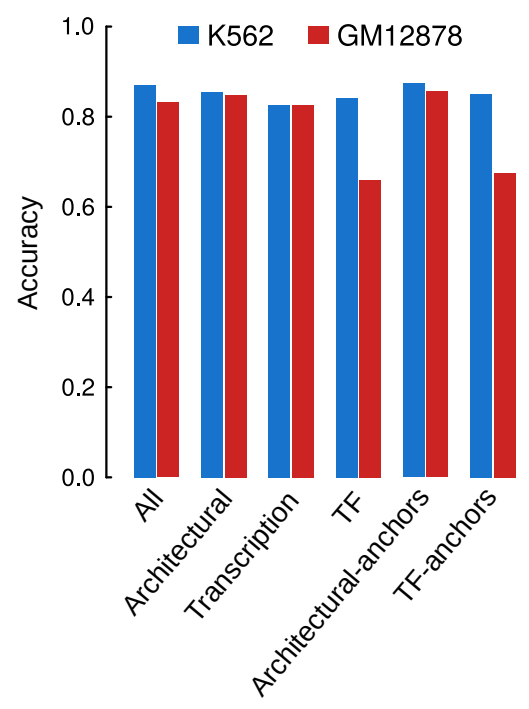

\section{Random forests}

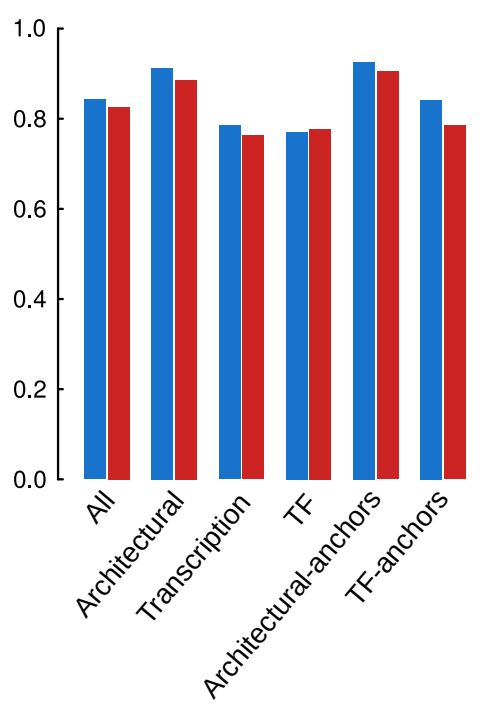

\section{XGBoost}

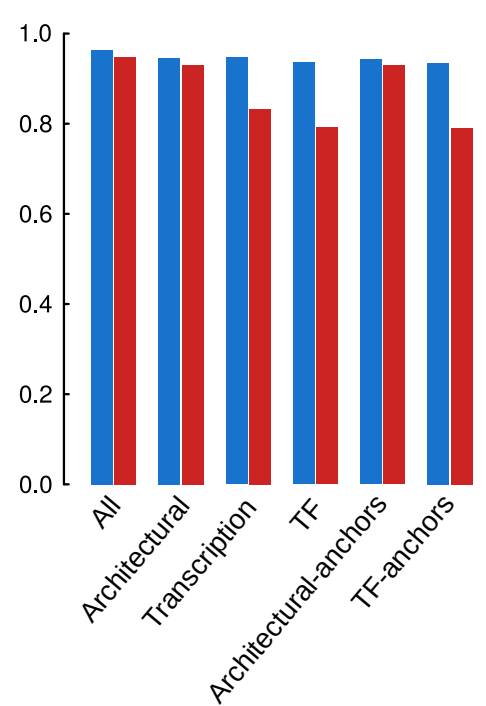

Figure 7. Accuracies of the DT, RF and XGBoost models trained with specific subset of chromatin features in K562 (blue) and GM12878 (red).

Unlike DT and XGBoost, RF performances seemed to be more consistent across cell lines and categories, with K562 achieving slightly better predictions (Figure 7, center panel; Tables 1, 2, 3, 4, S2-S9). Architectural associated categories yielded the best performances, with accuracies of 0.91-0.93 
bioRxiv preprint doi: https://doi.org/10.1101/2020.06.09.141473; this version posted June 10, 2020. The copyright holder for this preprint (which was not certified by peer review) is the author/funder. All rights reserved. No reuse allowed without permission.

and 0.89-0.91 in K562 and GM12878, respectively. It is worth to note that Architectural-anchors not only outperformed Architectural category, but also models trained with all the datasets (All) in both cell lines. This is also true for DT (Figure 7, left panel), and highlights the importance of information around anchors for the prediction of long-range chromatin interactions.

As we mentioned before, XGBoost yielded the best predictions (Figure 7, right panel) in both cell lines. In the case of K562, high accuracies were achieved independently of the selected features, confirming our observation that, at least in this cell line, not only architectural but also transcription factors are important determinants of RAD21-associated chromatin wiring.

\section{Conclusions}

3D genome organization plays a crucial role in the control of gene expression by allowing contacts between promoters and their distal regulatory partners. 3C-derived methods allow to perform chromatin architecture assays at the genome scale, but they remain laborious and expensive. Therefore, computational prediction based on publicly accessible sequencing datasets have emerged as an alternative strategy to generate virtual long range chromatin interaction maps for new cell types and conditions for which experimental maps are not yet available. Current approaches are hard to compare, since they differ both in the way chromatin interactions are modeled and the machine learning methods they use. Here, we use an integrative approach based on ENCODE 1D sequencing datasets to model long range chromatin interactions and test the performance of 5 state-of-the-art machine learning algorithms. We show that gradient boosting achieve the best predictions, yielding accuracies of $\sim 95 \%$. Moreover, we observe that chromatin features close to anchor regions contribute most to the predictions, in line with previous observations [15-17]. We also find that gradient boosting models trained with transcription-associated signals alone, unlike the other algorithms tested, are able to generate accurate predictions in K562 cell line, with transcription factors at the anchors being among the most predictive features. For this cell line, models trained with different subsets of features like architectural or transcription factors alone, are enough to produce accurate predictions.

In summary, our approach allows for systematic prediction of long-range chromatin interactions using publicly available 1D sequencing datasets. In light of our results, we propose gradient boosting as the best suited algorithm for this task. According to our data, XGBoost can be used to generate genome-wide maps of chromatin interactions, and information on few chromatin features at the anchors may be enough to yield accurate predictions. Analysis of interactions mediated by other proteins in different cell lines will help to clarify whether the observed performance for RAD21 mediated wiring in K562 can be generalized. In addition, prediction of de novo long-range chromatin maps genome-wide and subsequent comparisons with experimental data can be helpful to more comprehensively assess the predictive power of our strategy, as well as to exploit its full predictive potential.

Supplementary Materials: Figure S1: Chromatin features associated with RAD21 loops in K562 cell line. Panels are those described in Figure 1. Figure S2: Ranking of top 30 important features for the prediction of RAD21 chromatin loops. Horizontal bars represent relative importances as in Figure 3. Figure S3: Position specific importances of the whole set of sequencing datasets in K562 (A-C) and GM12878 (D-E) cell lines for DT (A,D), RF $(\mathrm{B}, \mathrm{E})$ and $\mathrm{XGBoost}(\mathrm{C}, \mathrm{F})$. Coordinates of the $\mathrm{x}$-axis are similar to those described in Figure 3. Figure S4: Ranking of top 30 important features for models trained without architectural factors information. Figure S5: Position specific importances of the whole set of sequencing datasets in K562 (A-C) and GM12878 (D-E) cell lines for DT $(\mathrm{A}, \mathrm{D}), \mathrm{RF}(\mathrm{B}, \mathrm{E})$ and $\mathrm{XGBoost}(\mathrm{C}, \mathrm{F})$. Models were trained without architectural factors information. Figure $\mathrm{S6}$ : Ranking of top 30 important features for models corresponding to the described categories: Architectural (A), $T F(B)$, Architectural-anchors (C) and TF-anchors (D). Table S1: Public data used in this study. Table S2: Performance of models trained with architectural factors binding information. K562 cell line. Table S3: Performance of models trained with architectural factors binding information. GM12878 cell line. Table S4: Performance of models trained with transcription factors binding information. K562 cell line. Table S5: Performance of models trained with transcription factors binding information. GM12878 cell line. Table S6: Performance of models trained with architectural factors binding information at loop anchors. K562 cell line. Table S7: Performance of models trained with architectural factors binding information at loop anchors. GM12878 cell line. Table S8: Performance of models trained with transcription factors binding information at loop anchors. K562 cell line. Table S9: Performance of models trained with transcription factors binding information at loop anchors. GM12878 cell line. 
bioRxiv preprint doi: https://doi.org/10.1101/2020.06.09.141473; this version posted June 10, 2020. The copyright holder for this preprint (which was not certified by peer review) is the author/funder. All rights reserved. No reuse allowed without permission.

Author Contributions: P.M.M.-G., F.D. and W.V. supervised the project. P.M.M.-G. conceived the method and designed the data analysis. T.V., P.M.M.-G. and M.G.-T. wrote the code. T.V., P.M.M.-G., F.D., M.G.-T. and F.G.-V. analyzed the data. T.V., P.M.M.-G., F.D., M.G.-T. and W.V. interpreted the results. T.V., P.M.M.-G., F.D. and M.G.-T. wrote the paper. All authors read, discussed and approved the manuscript.

Acknowledgements: We want to thank the Centro Informático Científico de Andalucía (CICA) for providing the high performance computing cluster, in which we performed all the analyses using custom scripts.

Conflicts of Interest: The authors declare no conflict of interest. The funders had no role in the design of the study; in the collection, analyses, or interpretation of data; in the writing of the manuscript, or in the decision to publish the results.

\section{References}

1. Bickmore, W.A.; Van Steensel, B. Genome architecture: Domain organization of interphase chromosomes, 2013. doi:10.1016/j.cell.2013.02.001.

2. Bonev, B.; Cavalli, G. Organization and function of the 3D genome, 2016. doi:10.1038/nrg.2016.112.

3. Rao, S.S.; Huntley, M.H.; Durand, N.C.; Stamenova, E.K.; Bochkov, I.D.; Robinson, J.T.; Sanborn, A.L.; Machol, I.; Omer, A.D.; Lander, E.S.; Aiden, E.L. A 3D map of the human genome at kilobase resolution reveals principles of chromatin looping. Cell 2014. doi:10.1016/j.cell.2014.11.021.

4. Weintraub, A.S.; Li, C.H.; Zamudio, A.V.; Sigova, A.A.; Hannett, N.M.; Day, D.S.; Abraham, B.J.; Cohen, M.A.; Nabet, B.; Buckley, D.L.; Guo, Y.E.; Hnisz, D.; Jaenisch, R.; Bradner, J.E.; Gray, N.S.; Young, R.A. YY1 Is a Structural Regulator of Enhancer-Promoter Loops. Cell 2017. doi:10.1016/j.cell.2017.11.008.

5. Dixon, J.R.; Selvaraj, S.; Yue, F.; Kim, A.; Li, Y.; Shen, Y.; Hu, M.; Liu, J.S.; Ren, B. Topological domains in mammalian genomes identified by analysis of chromatin interactions. Nature 2012, 485, 376-380. doi:10.1038/nature11082.

6. Nora, E.P.; Lajoie, B.R.; Schulz, E.G.; Giorgetti, L.; Okamoto, I.; Servant, N.; Piolot, T.; Van Berkum, N.L.; Meisig, J.; Sedat, J.; Gribnau, J.; Barillot, E.; Blüthgen, N.; Dekker, J.; Heard, E. Spatial partitioning of the regulatory landscape of the X-inactivation centre. Nature 2012. doi:10.1038/nature11049.

7. Lieberman-Aiden, E.; Van Berkum, N.L.; Williams, L.; Imakaev, M.; Ragoczy, T.; Telling, A.; Amit, I.; Lajoie, B.R.; Sabo, P.J.; Dorschner, M.O.; Sandstrom, R.; Bernstein, B.; Bender, M.A.; Groudine, M.; Gnirke, A.; Stamatoyannopoulos, J.; Mirny, L.A.; Lander, E.S.; Dekker, J. Comprehensive mapping of long-range interactions reveals folding principles of the human genome. Science 2009. doi:10.1126/science.1181369.

8. Zheng, H.; Xie, W. The role of 3D genome organization in development and cell differentiation, 2019. doi:10.1038/s41580-019-0132-4.

9. Li, Y.; Hu, M.; Shen, Y. Gene regulation in the 3D genome. Human molecular genetics 2018, 27, R228-R233. doi:10.1093/hmg/ddy164.

10. Schoenfelder, S.; Fraser, P. Long-range enhancer-promoter contacts in gene expression control, 2019. doi:10.1038/s41576-019-0128-0.

11. Dekker, J.; Marti-Renom, M.A.; Mirny, L.A. Exploring the three-dimensional organization of genomes: Interpreting chromatin interaction data, 2013. doi:10.1038/nrg3454.

12. Vian, L.; Pȩkowska, A.; Rao, S.S.; Kieffer-Kwon, K.R.; Jung, S.; Baranello, L.; Huang, S.C.; El Khattabi, L.; Dose, M.; Pruett, N.; Sanborn, A.L.; Canela, A.; Maman, Y.; Oksanen, A.; Resch, W.; Li, X.; Lee, B.; Kovalchuk, A.L.; Tang, Z.; Nelson, S.; Di Pierro, M.; Cheng, R.R.; Machol, I.; St Hilaire, B.G.; Durand, N.C.; Shamim, M.S.; Stamenova, E.K.; Onuchic, J.N.; Ruan, Y.; Nussenzweig, A.; Levens, D.; Aiden, E.L.; Casellas, R. The Energetics and Physiological Impact of Cohesin Extrusion. Cell 2018. doi:10.1016/j.cell.2018.03.072.

13. Huang, J.; Marco, E.; Pinello, L.; Yuan, G.C. Predicting chromatin organization using histone marks. Genome Biology 2015. doi:10.1186/s13059-015-0740-z.

14. Mourad, R.; Cuvier, O. Computational Identification of Genomic Features That Influence 3D Chromatin Domain Formation. PLoS Computational Biology 2016. doi:10.1371/journal.pcbi.1004908.

15. Kai, Y.; Andricovich, J.; Zeng, Z.; Zhu, J.; Tzatsos, A.; Peng, W. Predicting CTCF-mediated chromatin interactions by integrating genomic and epigenomic features. Nature Communications 2018. doi:10.1038/s41467-018-06664-6.

16. Al Bkhetan, Z.; Plewczynski, D. Three-dimensional Epigenome Statistical Model: Genome-wide Chromatin Looping Prediction. Scientific Reports 2018. doi:10.1038/s41598-018-23276-8. 
bioRxiv preprint doi: https://doi.org/10.1101/2020.06.09.141473; this version posted June 10, 2020. The copyright holder for this preprint (which was not certified by peer review) is the author/funder. All rights reserved. No reuse allowed without permission.

17. Zhang, S.; Chasman, D.; Knaack, S.; Roy, S. In silico prediction of high-resolution Hi-C interaction matrices. Nature Communications 2019. doi:10.1038/s41467-019-13423-8.

18. Consortium, E.P. An integrated encyclopedia of DNA elements in the human genome. Nature 2012, 489, 57-74. doi:10.1038/nature11247.

19. Handoko, L.; Xu, H.; Li, G.; Ngan, C.Y.; Chew, E.; Schnapp, M.; Lee, C.W.H.; Ye, C.; Ping, J.L.H.; Mulawadi, F.; Wong, E.; Sheng, J.; Zhang, Y.; Poh, T.; Chan, C.S.; Kunarso, G.; Shahab, A.; Bourque, G.; Cacheux-Rataboul, V.; Sung, W.K.; Ruan, Y.; Wei, C.L. CTCF-mediated functional chromatin interactome in pluripotent cells. Nature Genetics, 2011. doi:10.1038/ng.857.

20. Pedregosa, F.; Varoquaux, G.; Gramfort, A.; Michel, V.; Thirion, B.; Grisel, O.; Blondel, M.; Prettenhofer, P.; Weiss, R.; Dubourg, V.; Vanderplas, J.; Passos, A.; Cournapeau, D.; Brucher, M.; Perrot, M.; Duchesnay, E. Scikit-learn: Machine Learning in Python. Journal of Machine Learning Research 2011, 12, 2825-2830.

21. Buitinck, L.; Louppe, G.; Blondel, M.; Pedregosa, F.; Mueller, A.; Grisel, O.; Niculae, V.; Prettenhofer, P.; Gramfort, A.; Grobler, J.; Layton, R.; VanderPlas, J.; Joly, A.; Holt, B.; Varoquaux, G. API design for machine learning software: experiences from the scikit-learn project. ECML PKDD Workshop: Languages for Data Mining and Machine Learning, 2013, pp. 108-122.

22. Breiman, L. Random Forests. Machine Learning 2001, 45, 5-32.

23. Chen, T.; Guestrin, C. XGBoost: A Scalable Tree Boosting System. Proceedings of the 22Nd ACM SIGKDD International Conference on Knowledge Discovery and Data Mining; ACM: New York, NY, USA, 2016; KDD '16, pp. 785-794. doi:10.1145/2939672.2939785.

24. Haykin, S. Neural Networks: A Comprehensive Foundation, 2nd ed.; Prentice Hall PTR: Upper Saddle River, NJ, USA, 1998.

25. Hearst, M.A. Support Vector Machines. IEEE Intelligent Systems 1998, 13, 18-28. doi:10.1109/5254.708428.

26. Chang, Y.W.; Hsieh, C.J.; Chang, K.W.; Ringgaard, M.; Lin, C.J. Training and Testing Low-degree Polynomial Data Mappings via Linear SVM. Journal of Machine Learning Research 2010, 11(Apr), 14711490.

27. Powers, D.M.W. Evaluation: From precision, recall and f-measure to roc., informedness, markedness \& correlation. Journal of Machine Learning Technologies 2011, 2, 37-63.

28. Ivanov, D.; Nasmyth, K. A topological interaction between cohesin rings and a circular minichromosome. Cell 2005. doi:10.1016/j.cell.2005.07.018.

29. Arvey, A.; Agius, P.; Noble, W.S.; Leslie, C. Sequence and chromatin determinants of cell-type-specific transcription factor binding. Genome Research 2012. doi:10.1101/gr.127712.111.

30. Rockowitz, S.; Lien, W.H.; Pedrosa, E.; Wei, G.; Lin, M.; Zhao, K.; Lachman, H.M.; Fuchs, E.; Zheng, D. Comparison of REST Cistromes across Human Cell Types Reveals Common and Context-Specific Functions. PLoS Computational Biology 2014. doi:10.1371/journal.pcbi.1003671.

(C) 2020 by the authors. Submitted to bioRxiv for open access publication under the terms and conditions of the Creative Commons Attribution (CC BY) license (http:/ / creativecommons.org/licenses/by/4.0/). 